Article

\title{
Resilient Multiscale Coordination Control against Adversarial Nodes
}

\author{
Yilun Shang ${ }^{D}$ \\ School of Mathematical Sciences, Tongji University, Shanghai 200092, China; shyl@tongji.edu.cn; \\ Tel.: +86-6598-3240-2305
}

Received: 11 June 2018; Accepted: 12 July 2018; Published: 13 July 2018

\begin{abstract}
Multiscale consensus has been studied recently as a new concept in the field of multi-agent systems, which is able to accommodate many complicated coordination control tasks where values are measured in different scales due to, e.g., the constraints of physical environment. In this paper, we investigate the problem of resilient multiscale coordination control against a set of adversarial or non-cooperative nodes in directed networks. We design a multiscale filtering algorithm based upon local information which can withstand both faulty and Byzantine nodes. Building on the concept of network robustness, we establish necessary and sufficient conditions guaranteeing multiscale consensus with general time varying scales in the presence of globally bounded as well as locally bounded threats. In particular, for a network containing at most $\mathrm{R}$ faulty nodes, multiscale consensus is achieved if and only if the network is $(R+1, R+1)$-robust. The counterpart when having at most $\mathrm{R}$ Byzantine nodes instead is that the induced subnetwork of cooperative nodes is $R+1$-robust. Conditions guaranteeing resilient consensus for time-dependent networks are developed. Moreover, multiscale formation generation problems are introduced and solved as the generalizations. Finally, some numerical examples including applications in modular microgrids and power systems are worked out to demonstrate the availability of our theoretical results.
\end{abstract}

Keywords: consensus; multiscale coordination; time-varying scale; resilient consensus; network

\section{Introduction}

Coordination control over cooperative multi-agent net-works consisting of multiple individual subsystems has played a significant role in today's complex physical and engineered systems. A fundamental task of coordination control is reaching consensus collectively on global quantities of interest, such as the centroid of the network structure or the mean temperature of the environment, using only local information obtained by each agent in the network due to the limited communication capability [1]. Calculation and estimation through nearest neighbor interaction have been realized by a variety of consensus algorithms in the framework of distributed systems [2-4], where all individual agents can be measured by a unified scale enabling diverse applications such as traffic control, sensor networks, distributed optimization, and data aggregation.

In many physical-world applications, the states of agents tend to achieve agreement upon a certain consensus value but with different, possibly time-dependent, scales. For instance, in simultaneous coordination control of satellites running on orbits and their simulating robots on ground, prominent difference between the scales of vehicles' position and speed in space and on ground entails multi-scaled coordination control [5,6]. Other examples include water distribution systems, closed queuing networks, and compartmental mass-action systems [7]. As an extension to standard consensus, Roy [8] recently introduced a novel concept of scaled consensus, in which agents' states achieve asymptotically prescribed ratios in terms of multiple scales. It is noteworthy that the multiscale, or scaled, consensus offers a general basis for a wide range of consensus algorithms, which can 
be specialized to obtain standard consensus, cluster or group consensus [9], in which different subnetworks are allowed to achieve different consistent values, bipartite consensus [10] and sign consensus [11] by adopting appropriate scales.

Multiscale consensus has been explored for fixed strongly connected topology in [8] as well as switching topologies in [12], where the autonomous agents are described by continuous-time single integrators. Some scaled consensus protocols are proposed to solve the finite-time coordination control for continuous-time multi-vehicle systems in [13] and for discrete-time ones in [14]. In the recent work [15], multiscale consensus has been further characterized by sufficient and necessary conditions accommodating signal processing delays and signal transmission delays. All the aforementioned work assumes that all agents in the communication networks are cooperative. However, the performance of such systems deteriorates when one or more agents are compromised, potentially preventing the team of cooperative agents from achieving their goal. To the best of our knowledge, the problem of multiscale consensus in the presence of adversaries has not been addressed due to its complexity.

Large-scale cyber-physical systems are susceptible to adversarial or non-cooperative nodes due to malicious attacks (for instance, an attacker taking control of the communication module of certain agents trying to manipulate the entire network) or platform-level failures (for instance, a faulty robot sharing an incorrect location due to a defective Global Positioning System sensor). As such, resilience of consensus, and, more generally, information transmission, in the presence of adversarial nodes, has attracted significant attention in areas such as communication networks and mobile robotics over the past decades [16,17]. Recent remarkable efforts include a novel definition of network resilience by Zhang et al. and LeBlanc et al. [18,19], termed $r$-robustness, which facilitates purely local update rules for resilient consensus against malicious nodes. The advantage of the proposed approach is that the identities and actual number of non-cooperative nodes remain unknown to the cooperative nodes in the network. The results have later been generalized to the case of second-order multi-agent systems [20] and tolerance to communication delays is tackled in [21]. Furthermore, resilient flocking in time-dependent networks formed by mobile robot teams has been investigated in [22] and resilient opinion consensus in mobile social networks has been explored in [23]. It is worth mentioning that resilience to adversaries is conceptually distinct from resilience to disturbance or noise [24], and the methods used are very different.

Motivated by the aforementioned works, we in this paper consider the resilient multiscale coordination control for multi-agent systems in the presence of adversarial (faulty or malicious) nodes. The scope of the adversarial nodes is assumed to be either bounded by a constant in the whole network, which will be referred to as the globally bounded model, or by a constant in the neighborhood of each cooperative node, which will be referred to as the locally bounded model. The contribution of this paper is three-fold. First, compared with the previous resilient consensus results in e.g., [18-20], a generalization of the standard consensus protocol to multiscale consensus is proposed. We present sufficient and necessary conditions for multiscale consensus in time-invariant and time-varying networks that are modeled by directed graphs. Second, in the purview of multiscale consensus problems, arbitrary time-varying scales are addressed here, which largely extend the usual constant scales considered in e.g., [8,13-15] as well as the sign-preserving time-dependent scales studied in [12]. Finally, we introduce the multiscale resilient formation generation and formation tracking problems for both globally and locally bounded models in addition to resilient distributed estimation (i.e., multiscale consensus) as further generalizations, which are applicable in cooperative exploration and coordination tasks; e.g., $[25,26]$.

The remainder of the paper is organized as follows. Section 2 introduces the resilient multiscale consensus problems. The main results are given in Section 3. Simulation tests are performed to illustrate their effectiveness in Section 4 and conclusions are presented in Section 5. 


\section{Resilient Multiscale Consensus Algorithms}

\subsection{Graph Theory}

Let $\mathbb{R}$ and $\mathbb{N}$ be the sets of reals and non-negative integers, respectively. A time-dependent directed network (i.e., graph) over $n$ vertices is denoted by $\mathcal{G}(t)=(\mathcal{V}, \mathcal{E}(t))$, where $\mathcal{V}=\left\{v_{1}, \cdots, v_{n}\right\}$ represents the vertex set characterizing the agents in the network, and $\mathcal{E}(t) \subseteq \mathcal{V} \times \mathcal{V}$ represents the directed edge set at $t \in \mathbb{N}$. We consider a set partition of the vertex set as $\mathcal{V}=\mathcal{C} \cup \mathcal{A}$, in which $\mathcal{C}$ contains the set of cooperative nodes and $\mathcal{A}$ contains the set of adversarial nodes unknown a priori to the cooperative nodes. The information flow going from node $v_{i}$ to node $v_{j}$ is described by the edge $\left(v_{i}, v_{j}\right)$. The neighborhood of node $v_{i}$ at time $t$ is signified by $\mathcal{N}_{i}(t)=\left\{v_{j}:\left(v_{j}, v_{i}\right) \in\right.$ $\mathcal{E}(t)\}$. When considering that time-invariant networks are taken into consideration, we suppress the dependence on $t$ correspondingly. We sometimes do so in time-varying networks when no ambiguity will be caused within the context.

The following concepts of reachable sets and network robustness are introduced in [18], which have a close relationship with conventional graph-theoretic connectivity and play an essential role in resilient coordination $[19,27]$.

Definition 1 (reachable set). Suppose $r, s \in \mathbb{N}$. $S \subseteq \mathcal{V}$ is said to be an r-reachable set if there is a vertex $v_{i} \in S$ such that $\left|\mathcal{N}_{i} \backslash S\right| \geq r$. Moreover, $S$ is said to be an $(r, s)$-reachable set if $\left|\left\{v_{i} \in S:\left|\mathcal{N}_{i} \backslash S\right| \geq r\right\}\right| \geq s$. Clearly, r-reachability is equal to $(r, 1)$-reachability.

Definition 2 (network robustness). Let $r, s \in \mathbb{N}$. A digraph $\mathcal{G}$ is called r-robust if, for every pair of disjoint and nonempty subsets of $\mathcal{V}$, at least one of them is $r$-reachable. Furthermore, $\mathcal{G}$ is $(r, s)$-robust if for every pair of disjoint nonempty subsets $S_{1}, S_{2} \subseteq \mathcal{V}$, at least one of the following three statements holds: (i) $\left|\left\{v_{i} \in S_{1}:\left|\mathcal{N}_{i} \backslash S_{1}\right| \geq r\right\}\right|=\left|S_{1}\right|$, (ii) $\left|\left\{v_{i} \in S_{2}:\left|\mathcal{N}_{i} \backslash S_{2}\right| \geq r\right\}\right|=\left|S_{2}\right|$, and (iii) $\mid\left\{v_{i} \in S_{1}:\left|\mathcal{N}_{i} \backslash S_{1}\right| \geq\right.$ $r\}|+|\left\{v_{i} \in S_{2}:\left|\mathcal{N}_{i} \backslash S_{2}\right| \geq r\right\} \mid \geq s$.

\subsection{Model Description}

For a multi-agent system having the directed communication graph $\mathcal{G}(t)=(\mathcal{V}, \mathcal{E}(t))$ with $\mathcal{V}=\mathcal{C} \cup \mathcal{A}$, each cooperative node $v_{i} \in \mathcal{C}$ applies the following consensus update rule and communicates its value to its neighbors at every time-step $t \in \mathbb{N}$ :

$$
x_{i}(t+1)=f_{i}\left(\left\{x_{j}^{i}(t): v_{j} \in \mathcal{N}_{i}(t) \cup\left\{v_{i}\right\}\right\}\right)
$$

where $x_{j}^{i}(t) \in \mathbb{R}$ is the value sent from node $v_{j}$ to node $v_{i}$ at time $t$, and $x_{j}^{i}(t)=x_{j}(t)$ for $v_{j} \in \mathcal{C}$. Clearly, $x_{i}^{i}(t)=x_{i}(t)$. Here, $f_{i}$ delineates the update function for cooperative node $v_{i}$, which is to be designed so that the cooperative nodes could still reach the system's goal withstanding the compromise of adversarial nodes, whose identity and number remain unknown. Adversarial nodes, on the other hand, can apply arbitrary and different update rules that are not known to the cooperative nodes. We will consider the following two sorts of adversarial nodes.

Definition 3 (adversarial node). A node $v_{i} \in \mathcal{A}$ is faulty if it forwards value $x_{i}(t)$ to all of its neighbors at each time step, but implements some different update rule $f_{i}^{\prime}$ at some time step. A node $v_{i} \in \mathcal{A}$ is called Byzantine if it applies some different update rule $f_{i}^{\prime}$ or it fails to forward the same value to all the neighbors at some time step.

A faulty node is non-cooperative due to, for example, a faulty sensor or actuator, or intentional manipulation when the network is realized through broadcast communication. Byzantine nodes are often regarded as the worst-case attackers [19,21], who usually have a complete knowledge of the whole system and send information to its neighbors in a point-to-point way. Note that both faulty and 
Byzantine nodes can update their states arbitrarily at each time step, and that all faulty nodes are also Byzantine by definition, but not vice versa.

According to the location and number of the adversarial nodes, here we study the following two sorts of models: (i) R-globally bounded model, in which the number of nodes in $\mathcal{A}$ is bounded by a constant $\mathrm{R} \in \mathbb{N}$; and (ii) R-locally bounded model, in which $\left|\mathcal{N}_{i} \cap \mathcal{A}\right| \leq \mathrm{R}$ for every $v_{i} \in \mathcal{C}$. In the $\mathrm{R}$-locally bounded model, every cooperative node possesses no more than $\mathrm{R}$ adversarial neighbors. In both models, adversarial nodes threaten the group objective through stopping other agents from reaching valid states or driving their states into a dangerous range. Thus, strategies for resilient coordination are highly desirable.

\subsection{Multiscale Filtering Strategy}

Let $t \in \mathbb{N}$. Given a scalar scale $\alpha_{i}(t) \neq 0$ for agent $v_{i} \in \mathcal{V}$, we propose to deal with the multiscale consensus defined as follows.

Definition 4 (multiscale consensus). The multi-agent system (1) is said to achieve multiscale consensus with respect to $\left(\alpha_{1}(t), \cdots, \alpha_{n}(t)\right)$ if $\lim _{t \rightarrow \infty}\left(\alpha_{i}(t) x_{i}(t)-\alpha_{j}(t) x_{j}(t)\right)=0$ for any $v_{i}, v_{j} \in \mathcal{C}$ and any initial conditions $\left\{x_{i}(0)\right\}_{v_{i} \in \mathcal{V}}$.

It is obvious that multiscale consensus problems generalize standard consensus as well as cluster consensus problems [2,3]. It is also worth noting that Definition 4 admits general time-dependent scales, which are required restrictively to be invariant constants in [8,13-15] and sign-preserving in [12].

To accommodate the adversarial nodes in the network, we propose the following local filtering protocol, which extends the Weighted-Mean-Subsequence-Reduced (W-MSR) algorithm [18,19] for standard consensus. Specifically, the multiscale coordination consists of three steps, implemented at every time step $t$. Fix $R \in \mathbb{N}$. First, every cooperative node $v_{i} \in \mathcal{C}$ acquires the values $\left\{x_{j}^{i}(t)\right\}$ of its neighbors, and generates a decreasing list for $\left\{\alpha_{j}(t) x_{j}^{i}(t)\right\}$. Second, the largest $\mathrm{R}$ values which are strictly larger than $\alpha_{i}(t) x_{i}(t)$ in this sorted list are deleted (if there are fewer than $\mathrm{R}$ larger values than $\alpha_{i}(t) x_{i}(t)$, all of those values should be deleted). We apply a similar deletion process to the smaller values. The set of nodes that are deleted by node $v_{i}$ at time step $t$ is denoted by $\mathcal{R}_{i}(t)$. Third, each $v_{i} \in \mathcal{C}$ updates its value using the following rule:

$$
x_{i}(t+1)=\operatorname{sgn}\left(\alpha_{i}(t)\right) \sum_{v_{j} \in\left(\mathcal{N}_{i}(t) \cup\left\{v_{i}\right\}\right) \backslash \mathcal{R}_{i}(t)} w_{i j}(t) \alpha_{j}(t) x_{j}^{i}(t),
$$

where $\operatorname{sgn}(\cdot)$ is the signum function, and $w_{i j}(t)$ are the weights satisfying (i) $w_{i j}(t)=0$ if $v_{j} \notin$ $\mathcal{N}_{i}(t) \cup\left\{v_{i}\right\}$, (ii) there is some constant $\alpha \in(0,1)$ independent of $t$, such that $w_{i j}(t)\left|\alpha_{i}(t)\right| \geq \alpha>0$ for any $v_{j} \in\left(\mathcal{N}_{i}(t) \cup\left\{v_{i}\right\}\right) \backslash \mathcal{R}_{i}(t)$, and (iii) $\sum_{v_{j} \in\left(\mathcal{N}_{i}(t) \cup\left\{v_{i}\right\}\right) \backslash \mathcal{R}_{i}(t)} w_{i j}(t)\left|\alpha_{i}(t)\right|=1$.

Notice that the above weights $w_{i j}(t)$ may be arbitrarily selected provided these three conditions are true. The algorithmic complexity is low and only local information is utilized. Hence, the algorithm can be regarded as purely distributed. No prior knowledge of the identities of adversarial nodes or the network topology is assumed for cooperative nodes. The above algorithm will be referred to as the multiscale filtering strategy with parameter $\mathrm{R}$.

\section{Consensus Analysis}

Here, we present the consensus analysis for the multi-agent system in the presence of both faulty and Byzantine nodes. In every case, we study resilience results for both globally bounded threats and locally bounded threats. To begin with, set $M(t):=\max _{v_{i} \in \mathcal{C}}\left\{\alpha_{i}(t) x_{i}(t)\right\}$ and $m(t):=$ $\min _{v_{i} \in \mathcal{C}}\left\{\alpha_{i}(t) x_{i}(t)\right\}$. The update rule in (2) implies that 


$$
\alpha_{i}(t+1) x_{i}(t+1)=\left|\alpha_{i}(t)\right| \sum_{j \in\left(\mathcal{N}_{i}(t) \cup\left\{v_{i}\right\}\right) \backslash \mathcal{R}_{i}(t)} w_{i j}(t) \alpha_{j}(t) x_{j}^{i}(t),
$$

which is a convex combination of $\left\{\alpha_{j}(t) x_{j}^{i}(t)\right\}_{j \in\left(\mathcal{N}_{i}(t) \cup\left\{v_{i}\right\}\right) \backslash \mathcal{R}_{i}(t)}$. It is straightforward to see that, for all $i \in \mathcal{C}$ and $t \in \mathbb{N}, \alpha_{i}(t+1) x_{i}(t+1) \in[m(t), M(t)]$ holds in both R-globally and R-locally bounded models in the presence of faulty/Byzantine nodes when the multiscale filtering strategy with parameter $\mathrm{R}$ is applied. This means the interval $[m(0), M(0)]$ is always an invariant set in the sense that the scaled values of cooperative nodes remain in this range for all $t$.

\subsection{Multiscale Coordination in the Presence of Faulty Nodes}

In the following, we establish some necessary and sufficient conditions for multiscale coordination in the globally/locally bounded model with faulty nodes. We consider time-invariant networks first, and then extend the results to time-dependent networks. The proofs are based on the techniques in [19].

Theorem 1 (consensus in globally bounded model with faulty nodes). Consider a time-invariant network characterized by a digraph $\mathcal{G}=(\mathcal{V}, \mathcal{E})$, where every cooperative node updates its value according to the multiscale filtering strategy with parameter $\mathrm{R}$. Therefore, in the R-globally bound model having faulty nodes, multiscale consensus is reached if and only if $\mathcal{G}$ is $(R+1, R+1)$-robust.

Proof. (Necessity) Assume that $\mathcal{G}$ is not an $(\mathrm{R}+1, \mathrm{R}+1)$-robust graph. Thus, there are disjoint nonempty sets $S_{1}, S_{2} \subseteq \mathcal{V}$ such that none of the statements (i)-(iii) in Definition 2 hold. Fix $a<b$. Let $x_{i}(0)=\frac{a}{\alpha_{i}(0)}$ for any node $v_{i} \in S_{1}$, and $x_{i}(0)=\frac{b}{\alpha_{i}(0)}$ for any node $v_{i} \in S_{2}$. For $v_{i} \in \mathcal{V} \backslash\left\{S_{1} \cup S_{2}\right\}$, set $x_{i}(0)=\frac{c}{\alpha_{i}(0)}$ for some fixed $c \in(a, b)$. Since $\mid\left\{v_{i} \in S_{1}:\left|\mathcal{N}_{i} \backslash S_{1}\right| \geq\right.$ $\mathrm{R}+1\}|+|\left\{\mathrm{v}_{\mathrm{i}} \in \mathrm{S}_{2}:\left|\mathcal{N}_{\mathrm{i}} \backslash \mathrm{S}_{2}\right| \geq \mathrm{R}+1\right\} \mid \leq \mathrm{R}$, suppose that all nodes in $\left\{v_{i} \in S_{1}:\left|\mathcal{N}_{i} \backslash S_{1}\right| \geq \mathrm{R}+1\right\}$ and $\left\{v_{i} \in S_{2}:\left|\mathcal{N}_{i} \backslash S_{2}\right| \geq \mathrm{R}+1\right\}$ are faulty nodes and that they keep their values unchanged. Since there is at least one cooperative node in both $S_{1}$ and $S_{2}$ (because $\left|\left\{v_{i} \in S_{1}:\left|\mathcal{N}_{i} \backslash S_{1}\right| \geq \mathrm{R}+1\right\}\right|<\left|S_{1}\right|$ and $\left.\left|\left\{v_{i} \in S_{2}:\left|\mathcal{N}_{i} \backslash S_{2}\right| \geq \mathrm{R}+1\right\}\right|<\left|\mathrm{S}_{2}\right|\right)$, the scaled values $\alpha_{i}(t) x_{i}(t)$ of such cooperative nodes cannot reach consensus by using the multiscale filtering strategy with parameter $R$. Therefore, multiscale consensus can not be achieved.

(Sufficiency) From the comments in the beginning of this section, we may assume that $\rho_{M}:=$ $\lim _{t \rightarrow \infty} M(t) \geq \rho_{m}:=\lim _{t \rightarrow \infty} m(t)$. If $\rho_{M}=\rho_{m}$, multiscale consensus is then reached. In what follows, we assume that $\rho_{M}>\rho_{m}$ and will show that it does not hold by using contradiction.

Choose $\varepsilon_{0}>0$ satisfying $\rho_{M}-\varepsilon_{0}>\rho_{m}+\varepsilon_{0}$. For $t \in \mathbb{N}$ and $\varepsilon_{k}>0$, we define two sets $A_{M}\left(t, \varepsilon_{k}\right):=\left\{v_{i} \in \mathcal{V}: \alpha_{i}(t) x_{i}(t)>\rho_{M}-\varepsilon_{k}\right\}$ and $A_{m}\left(t, \varepsilon_{k}\right):=\left\{v_{i} \in \mathcal{V}: \alpha_{i}(t) x_{i}(t)<\rho_{m}+\varepsilon_{k}\right\}$. By the definition of $\varepsilon_{0}, A_{M}\left(t, \varepsilon_{0}\right)$ and $A_{m}\left(t, \varepsilon_{0}\right)$ are disjoint. Fix $\varepsilon<\frac{\alpha^{|\mathcal{C}|} \varepsilon_{0}}{1-\alpha|\mathcal{C}|}$ and $\varepsilon_{0}>\varepsilon>0$. Let $t_{\varepsilon}$ be the time step satisfying $M(t)<\rho_{M}+\varepsilon$ and $m(t)>\rho_{m}-\varepsilon$ for all $t \geq t_{\varepsilon}$.

Recall that $A_{M}\left(t_{\varepsilon}, \varepsilon_{0}\right)$ and $A_{m}\left(t_{\varepsilon}, \varepsilon_{0}\right)$ are nonempty disjoint sets. Noting that $\mathcal{G}$ is $(\mathrm{R}+1, \mathrm{R}+1)$-robust with at most $\mathrm{R}$ faulty nodes, there must be a cooperative node in their union which contains more than or a number equal to $\mathrm{R}+1$ neighbors outside of its set. We suppose, without loss of generality, that $v_{i} \in A_{M}\left(t_{\varepsilon}, \varepsilon_{0}\right) \cap \mathcal{C}$ has more than or equal to $\mathrm{R}+1$ neighbors outside of $A_{M}\left(t_{\varepsilon}, \varepsilon_{0}\right)$. Since these neighbors' values are at most equal to $\rho_{M}-\varepsilon_{0}$ and at least one of these values will be used by $v_{i}$, we obtain

$$
\begin{aligned}
\alpha_{i}\left(t_{\varepsilon}+1\right) x_{i}\left(t_{\varepsilon}+1\right) & \leq(1-\alpha) M\left(t_{\varepsilon}\right)+\alpha\left(\rho_{M}-\varepsilon_{0}\right) \\
& \leq \rho_{M}-\alpha \varepsilon_{0}+(1-\alpha) \varepsilon
\end{aligned}
$$

where we have used the inequality $M\left(t_{\varepsilon}\right) \leq \rho_{M}+\varepsilon$, and the fact that each cooperative node's value is a convex combination of its own value and the values of its neighbors with coefficients larger than or equal to $\alpha$ and that the largest value $v_{i}$ will use at time $t_{\varepsilon}$ no more than $M\left(t_{\varepsilon}\right)$ according to the multiscale filtering strategy with parameter R. The expression (4) also applies to the updated value of 
any cooperative node outside $A_{M}\left(t_{\varepsilon}, \varepsilon_{0}\right)$ since such a node will adopt its own value (which is also less than or equal to $\left.\rho_{M}-\varepsilon_{0}\right)$ in the update process. Likewise, if $v_{i} \in A_{m}\left(t_{\varepsilon}, \varepsilon_{0}\right) \cap \mathcal{C}$, which has more than or a number equal to $\mathrm{R}+1$ neighbors outside of $A_{m}\left(t_{\varepsilon}, \varepsilon_{0}\right)$, we derive a similar inequality

$$
\alpha_{i}\left(t_{\varepsilon}+1\right) x_{i}\left(t_{\varepsilon}+1\right) \geq \rho_{m}+\alpha \varepsilon_{0}-(1-\alpha) \varepsilon,
$$

which also applies to the cooperative nodes outside $A_{m}\left(t_{\varepsilon}, \varepsilon_{0}\right)$.

Now, define $\varepsilon_{1}=\alpha \varepsilon_{0}-(1-\alpha) \varepsilon$ and let $0<\varepsilon<\varepsilon_{1}<\varepsilon_{0}$. Notice that the sets $A_{M}\left(t_{\varepsilon}+1, \varepsilon_{1}\right)$ and $A_{m}\left(t_{\varepsilon}+1, \varepsilon_{1}\right)$ are disjoint. The discussion in the above paragraph implies that $\mid A_{M}\left(t_{\varepsilon}+1, \varepsilon_{1}\right) \cap$ $\mathcal{C}|<| A_{M}\left(t_{\varepsilon}, \varepsilon_{0}\right) \cap \mathcal{C} \mid$ or $\left|A_{m}\left(t_{\varepsilon}+1, \varepsilon_{1}\right) \cap \mathcal{C}\right|<\left|A_{m}\left(t_{\varepsilon}, \varepsilon_{0}\right) \cap \mathcal{C}\right|$ holds. We can recursively define $\varepsilon_{k}=\alpha \varepsilon_{k-1}-(1-\alpha) \varepsilon$ for each $k \geq 1$ and note that $\varepsilon_{k}<\varepsilon_{k-1}$. The above comments can be applied to every time step $t_{\varepsilon}+k$ provided there still exist cooperative nodes in $A_{M}\left(t_{\varepsilon}+k, \varepsilon_{k}\right)$ and $A_{m}\left(t_{\varepsilon}+k, \varepsilon_{k}\right)$. Since there are $|\mathcal{C}|$ cooperative nodes in the entire graph, there is some $T \leq|\mathcal{C}|$ such that either $A_{M}\left(t_{\varepsilon}+T, \varepsilon_{T}\right) \cap \mathcal{C}$ or $A_{m}\left(t_{\varepsilon}+T, \varepsilon_{T}\right) \cap \mathcal{C}$ is empty. However, $\varepsilon_{T}=\alpha \varepsilon_{T-1}-(1-\alpha) \varepsilon=\alpha^{T} \varepsilon_{0}-(1-$ $\left.\alpha^{T}\right) \varepsilon \geq \alpha^{|\mathcal{C}|} \varepsilon_{0}-\left(1-\alpha^{|\mathcal{C}|}\right) \varepsilon>0$ as per the choice of $\varepsilon$. This means that every cooperative node at time $t_{\varepsilon}+T$ has a value of no more than $\rho_{M}-\varepsilon_{T}<\rho_{M}$ or has a value of at least $\rho_{m}+\varepsilon_{T}>\rho_{m}$. This contradicts the definition of $\rho_{M}$ or $\rho_{m}$. The result then follows.

For time-dependent networks, we show the following corollary.

Corollary 1. Consider a time-varying network characterized by a digraph $\mathcal{G}(t)=(\mathcal{V}, \mathcal{E}(t))$, where every cooperative node updates its value according to the multiscale filtering strategy with parameter $\mathrm{R}$. Denote by $\left\{t_{k}\right\}$ the time steps in which $\mathcal{G}(t)$ is $(\mathrm{R}+1, \mathrm{R}+1)$-robust. Therefore, in the $\mathrm{R}$-globally bound model with faulty nodes, multiscale consensus is reached if $\left|\left\{t_{k}\right\}\right|=\infty$ and there is a constant $c$ such that $\left|t_{k+1}-t_{k}\right| \leq c$ for all $k$.

Proof. We proceed similarly as in Theorem 1. We now set $\varepsilon<\frac{\alpha^{|\mathcal{C}|} \mid \varepsilon_{0}}{1-\alpha^{|\mathcal{C}| c}}$ and $\varepsilon_{0}>\varepsilon>0$. Define $t_{\varepsilon}$ to be the time step satisfying $M(t)<\rho_{M}+\varepsilon$ and $m(t)>\rho_{m}-\varepsilon$ for all $t \geq t_{\varepsilon}$. By assumption, there is $\tau_{1} \in\left\{t_{\varepsilon}, t_{\varepsilon}+1, \cdots, t_{\varepsilon}+c-1\right\}$ such that $\mathcal{G}\left(\tau_{1}\right)$ is $(\mathrm{R}+1, \mathrm{R}+1)$-robust. Let $\varepsilon_{1}=\alpha \varepsilon_{0}-(1-\alpha) \varepsilon$ and $0<\varepsilon<\varepsilon_{1}<\varepsilon_{0}$. Reasoning as in the proof of Theorem 1, we see that $\left|A_{M}\left(\tau_{1}+1, \varepsilon_{1}\right) \cap \mathcal{C}\right|<$ $\left|A_{M}\left(\tau_{1}, \varepsilon_{0}\right) \cap \mathcal{C}\right|$ or $\left|A_{m}\left(\tau_{1}+1, \varepsilon_{1}\right) \cap \mathcal{C}\right|<\left|A_{m}\left(\tau_{1}, \varepsilon_{0}\right) \cap \mathcal{C}\right|$ holds.

We can recursively define $\varepsilon_{k}=\alpha \varepsilon_{k-1}-(1-\alpha) \varepsilon$ for $1 \leq k \leq|\mathcal{C}| c$. Similarly as in Theorem 1 , we can prove that every cooperative node $v_{i}$ satisfying $\alpha_{i}\left(\tau_{1}+1\right) x_{i}\left(\tau_{1}+1\right) \leq \rho_{M}-\varepsilon_{1}$ will satisfy $\alpha_{i}\left(\tau_{1}+k\right) x_{i}\left(\tau_{1}+k\right) \leq \rho_{M}-\varepsilon_{k}$ for each $1 \leq k \leq|\mathcal{C}| c$. In the same manner, every cooperative node $v_{i}$ satisfying $\alpha_{i}\left(\tau_{1}+1\right) x_{i}\left(\tau_{1}+1\right) \geq \rho_{m}+\varepsilon_{1}$ will satisfy $\alpha_{i}\left(\tau_{1}+k\right) x_{i}\left(\tau_{1}+k\right) \geq \rho_{m}+\varepsilon_{k}$ for each $1 \leq k \leq|\mathcal{C}| c$. Therefore, we have $\left|A_{M}\left(\tau_{1}+k, \varepsilon_{k}\right) \cap \mathcal{C}\right| \leq\left|A_{M}\left(\tau_{1}+k-1, \varepsilon_{k-1}\right) \cap \mathcal{C}\right|$ or $\mid A_{m}\left(\tau_{1}+\right.$ $\left.k, \varepsilon_{k}\right) \cap \mathcal{C}|\leq| A_{m}\left(\tau_{1}+k-1, \varepsilon_{k-1}\right) \cap \mathcal{C} \mid$ for every $1 \leq k \leq|\mathcal{C}| c$ irrespective of the graph structure. By assumption, there exists an infinite sequence of $\tau_{1}, \tau_{2}, \cdots$ where $\mathcal{G}\left(\tau_{k}\right)$ is $(\mathrm{R}+1, \mathrm{R}+1)$-robust, both $A_{M}\left(\tau_{k}, \varepsilon_{\tau_{k}-\tau_{1}}\right) \cap \mathcal{C}$ and $A_{m}\left(\tau_{k}, \varepsilon_{\tau_{k}-\tau_{1}}\right) \cap \mathcal{C}$ are non-empty, and either $\left|A_{M}\left(\tau_{k}+1, \varepsilon_{\tau_{k}+1-\tau_{1}}\right) \cap \mathcal{C}\right|<$ $\left|A_{M}\left(\tau_{k}, \varepsilon_{\tau_{k}-\tau_{1}}\right) \cap \mathcal{C}\right|$ or $\left|A_{m}\left(\tau_{k}+1, \varepsilon_{\tau_{k}+1-\tau_{1}}\right) \cap \mathcal{C}\right|<\left|A_{m}\left(\tau_{k}, \varepsilon_{\tau_{k}-\tau_{1}}\right) \cap \mathcal{C}\right|$, or both, holds for $k \geq 1$. As there exist $|\mathcal{C}|$ cooperative nodes in the entire graph and $\left|\tau_{|\mathcal{C}|}-\tau_{1}\right| \leq|\mathcal{C}| c$, there is some $T \leq|\mathcal{C}| \mathcal{C}$ such that either $A_{M}\left(\tau_{1}+T, \varepsilon_{T}\right) \cap \mathcal{C}$ or $A_{m}\left(\tau_{1}+T, \varepsilon_{T}\right) \cap \mathcal{C}$ is empty. Recalling the definition of $\varepsilon$, we obtain $\varepsilon_{T}>0$. This gives rise to a contradiction similarly as in the proof of Theorem 1 .

Next, we extend the above results to solve resilient formation generation problems for multi-agent systems in the presence of adversarial nodes. In standard formation generation problems, the aim is to design distributed protocols to guarantee that each pair of neighboring agents reach a desired relative position with respect to each other $[3,4,28]$. A certain pattern is thus formed by the agents as a whole. In the context of resilient multiscale coordination, we introduce the multiscale formation generation as follows. 
Definition 5 (multiscale formation generation). Let $g=\left(g_{1}, \cdots, g_{n}\right) \in \mathbb{R}^{n}$. The agents in $\mathcal{G}$ are said to achieve the multiscale formation $g$ with respect to $\left(\alpha_{1}(t), \cdots, \alpha_{n}(t)\right)$ if $\lim _{t \rightarrow \infty}\left(\alpha_{i}(t) x_{i}(t)-\alpha_{j}(t) x_{j}(t)\right)=$ $g_{i}-g_{j}$ for all $v_{i}, v_{j} \in \mathcal{C}$ and all initial conditions $\left\{x_{i}(0)\right\}_{v_{i} \in \mathcal{V}}$.

It is easy to see that the agents reach the multiscale formation $g$ if there exists a vector $h \in \mathbb{R}^{n}$ such that for $v_{i} \in \mathcal{C}, \alpha_{i}(t) x_{i}(t)$ tends to $g_{i}+h$ as time goes to infinity. To this end, we design the following formation generation rule for each $v_{i} \in \mathcal{C}$ :

$$
x_{i}(t+1)=\frac{1}{\alpha_{i}(t)} g_{i}+\operatorname{sgn}\left(\alpha_{i}(t)\right) \cdot \sum_{v_{j} \in\left(\mathcal{N}_{i}(t) \cup\left\{v_{i}\right\}\right) \backslash \mathcal{R}_{i}(t)} w_{i j}(t)\left(\alpha_{j}(t) x_{j}^{i}(t)-g_{j}\right),
$$

where the weights $w_{i j}(t)$ satisfy the same conditions in Section 2.3. In the formation control problem, we will modify the three-step multiscale filtering strategy with parameter $\mathrm{R}$ introduced in Section 2.3 in three places: first, the sorted list is created for $\left\{\alpha_{j}(t) x_{j}^{i}(t)-g_{j}\right\}$ instead of $\left\{\alpha_{j}(t) x_{j}^{i}(t)\right\}$. Second, the largest $\mathrm{R}$ values that are strictly greater than $\alpha_{i}(t) x_{i}(t)-g_{i}$ instead of $\alpha_{i}(t) x_{i}(t)$ in the list are deleted. The same modification applies to the smaller values. Third, the update rule (2) is replaced by (6). We shall refer to this modified algorithm as the multiscale filtering-formation strategy with parameter $R$.

Corollary 2 (formation generation in globally bounded model with faulty nodes). Consider a time-invariant graph characterized by a digraph $\mathcal{G}=(\mathcal{V}, \mathcal{E})$, where every cooperative node updates its value according to the multiscale filtering-formation strategy with parameter $\mathrm{R}$. Therefore, in the R-globally bounded model having faulty nodes, multiscale formation $g$ is reached if and only if $\mathcal{G}$ is $(R+1, R+1)$-robust.

For a time-dependent network $\mathcal{G}(t)=(\mathcal{V}, \mathcal{E}(t))$, let $\left\{t_{k}\right\}$ be the time steps where $\mathcal{G}(t)$ is $(\mathrm{R}+1, \mathrm{R}+1)$-robust. Therefore, in the $\mathrm{R}$-globally bounded model having faulty nodes, multiscale formation $\mathrm{g}$ is reached if $\left|\left\{t_{k}\right\}\right|=\infty$ and there is a constant $c$ satisfying $\left|t_{k+1}-t_{k}\right| \leq c$ for every $k$.

Proof. Let $\bar{x}_{j}^{i}(t)=x_{j}^{i}(t)-g_{j} / \alpha_{j}(t)$ and $\bar{x}_{j}(t)=x_{j}(t)-g_{j} / \alpha_{j}(t)$ for $v_{i}, v_{j} \in \mathcal{V}$. Then, the update rule (6) becomes $\bar{x}_{i}(t+1)=\operatorname{sgn}\left(\alpha_{i}(t)\right) \sum_{j \in\left(\mathcal{N}_{i}(t) \cup\left\{v_{i}\right\}\right) \backslash \mathcal{R}_{i}(t)} w_{i j}(t) \alpha_{j}(t) \bar{x}_{j}^{i}(t)$ for $v_{i} \in \mathcal{C}$.

For time-invariant network topology, it follows from Theorem 1 that $\mathcal{G}$ is $(\mathrm{R}+1, \mathrm{R}+1)$-robust if and only if multiscale consensus is achieved for $\left\{\bar{x}_{i}(t)\right\}_{v_{i} \in \mathcal{C}}$, which in turn is equivalent to having a vector $h \in \mathbb{R}^{n}$ such that $\lim _{t \rightarrow \infty} \alpha_{i}(t) x_{i}(t)=g_{i}+h$. The result then follows. In the case of time-varying topology, a similar argument applies by virtue of Corollary 1.

For locally bounded models, where adversarial nodes are much more popular but the number of them are still bounded in every cooperative node's neighborhood, we propose to characterize the network structure that is ideal for coping with fault nodes as follows.

Theorem 2 (consensus in locally bounded model with faulty nodes). Consider a time-invariant network characterized by a digraph $\mathcal{G}=(\mathcal{V}, \mathcal{E})$, in which every cooperative node updates its value following the multiscale filtering strategy with parameter R. Therefore, in the R-locally bound model having faulty nodes, multiscale consensus is achieved if $\mathcal{G}$ is $2 \mathrm{R}+1$-robust. Moreover, $\mathcal{G}$ is $\mathrm{R}+1$-robust if multiscale consensus in the R-locally bound model with faulty nodes is achieved.

Proof. (Necessity) Assume that $\mathcal{G}$ is not $\mathrm{R}+1$-robust. Therefore, there exist disjoint nonempty sets $S_{1}, S_{2} \subseteq \mathcal{V}$ such that every node in these two sets will have at most $\mathrm{R}$ neighbors outside the set. Suppose that there exist cooperative nodes in both $S_{1}$ and $S_{2}$. Fix $a<b$. Let $x_{i}(0)=\frac{a}{\alpha_{i}(0)}$ for any node $v_{i} \in S_{1}$, and $x_{i}(0)=\frac{b}{\alpha_{i}(0)}$ for any node $v_{i} \in S_{2}$. For $v_{i} \in \mathcal{V} \backslash\left\{S_{1} \cup S_{2}\right\}$, set $x_{i}(0)=\frac{c}{\alpha_{i}(0)}$ for some fixed $c \in(a, b)$. Clearly, the scaled values $\alpha_{i}(t) x_{i}(t)$ of nodes in $S_{1}$ and $S_{2}$ will not achieve consensus under the multiscale filtering strategy with parameter $\mathrm{R}$ because they will not adopt any values from outside their own sets. Thus, multiscale consensus can not be reached. 
(Sufficiency) We proceed similarly as in Theorem 1. Suppose that $\rho_{M}:=\lim _{t \rightarrow \infty} M(t)$ and $\rho_{m}:=$ $\lim _{t \rightarrow \infty} m(t)$. In what follows, we will prove $\rho_{M}=\rho_{m}$ by contradiction. For this purpose, assume that $\rho_{M}>\rho_{m}$. Choose $\varepsilon_{0}>0$ so that $\rho_{M}-\varepsilon_{0}>\rho_{m}+\varepsilon_{0}$. For $t \in \mathbb{N}$ and $\varepsilon_{k}>0$, we consider two sets given by $A_{M}\left(t, \varepsilon_{k}\right):=\left\{v_{i} \in \mathcal{V}: \alpha_{i}(t) x_{i}(t)>\rho_{M}-\varepsilon_{k}\right\}$ and $A_{m}\left(t, \varepsilon_{k}\right):=\left\{v_{i} \in \mathcal{V}: \alpha_{i}(t) x_{i}(t)<\rho_{m}+\varepsilon_{k}\right\}$. By the definition of $\varepsilon_{0}, A_{M}\left(t, \varepsilon_{0}\right)$ and $A_{m}\left(t, \varepsilon_{0}\right)$ are disjoint. Set $\varepsilon<\frac{\alpha^{|\mathcal{C}|} \varepsilon_{0}}{1-\alpha|\mathcal{C}|}$ and $\varepsilon_{0}>\varepsilon>0$. Define $t_{\varepsilon}$ to be the time step satisfying $M(t)<\rho_{M}+\varepsilon$ and $m(t)>\rho_{m}-\varepsilon$ for any $t \geq t_{\varepsilon}$.

Recall that the two sets $A_{M}\left(t_{\varepsilon}, \varepsilon_{0}\right) \cap \mathcal{C}$ and $A_{m}\left(t_{\varepsilon}, \varepsilon_{0}\right) \cap \mathcal{C}$ are nonempty and disjoint. Since $\mathcal{G}$ is $2 \mathrm{R}+1$-robust, one of these two sets must be $2 \mathrm{R}+1$-reachable if not both. We suppose, without loss of generality, that $A_{M}\left(t_{\varepsilon}, \varepsilon_{0}\right) \cap \mathcal{C}$ is $2 \mathrm{R}+1$-reachable, and hence there exists a node $v_{i} \in A_{M}\left(t_{\varepsilon}, \varepsilon_{0}\right) \cap \mathcal{C}$ which has no less than $2 \mathrm{R}+1$ neighboring nodes outside its set. Because there exist no more than $\mathrm{R}$ faulty nodes in $\mathcal{N}_{i}, v_{i}$ will adopt no less than one of its cooperative neighbors' values outside $A_{M}\left(t_{\varepsilon}, \varepsilon_{0}\right) \cap \mathcal{C}$ under the multiscale filtering strategy with parameter $\mathrm{R}$. Consequently, proceeding as in the proof of Theorem 1, we derive $\alpha_{i}\left(t_{\varepsilon}+1\right) x_{i}\left(t_{\varepsilon}+1\right) \leq \rho_{M}-\alpha \varepsilon_{0}+(1-\alpha) \varepsilon$. This also holds true for the renewed value of every cooperative node outside $A_{M}\left(t_{\varepsilon}, \varepsilon_{0}\right) \cap \mathcal{C}$ as such a node will adopt its own value in the renewal procedure. Analogously, if $v_{i} \in A_{m}\left(t_{\varepsilon}, \varepsilon_{0}\right) \cap \mathcal{C}$ has more than or equal to $2 \mathrm{R}+1$ neighbors outside its set, we have a similar bound $\alpha_{i}\left(t_{\varepsilon}+1\right) x_{i}\left(t_{\varepsilon}+1\right) \geq \rho_{m}+\alpha \varepsilon_{0}-(1-\alpha) \varepsilon$, which also applies to the cooperative nodes outside $A_{m}\left(t_{\varepsilon}, \varepsilon_{0}\right) \cap \mathcal{C}$. Now by defining $\varepsilon_{1}=\alpha \varepsilon_{0}-(1-\alpha) \varepsilon$ which satisfies $0<\varepsilon<\varepsilon_{1}<\varepsilon_{0}$, we can use the same proof in Theorem 1 , by setting recursively $\varepsilon_{k}$, for $k \geq 1$ to produce the contradiction. We then proved the sufficiency part.

Note that there is a gap between the sufficient condition and the necessary condition in Theorem 2. In fact, the example mentioned in ([19], Figure 4) can also be used to show that the sufficient condition here is sharp. In the case of time-dependent networks, we show the following result, which more or less can be shown in the same way as Corollary 1.

Corollary 3. Given a time-dependent network characterized by a digraph $\mathcal{G}(t)=(\mathcal{V}, \mathcal{E}(t))$, where every cooperative node updates its value following the multiscale filtering strategy with parameter $\mathrm{R}$. Let $\left\{t_{k}\right\}$ be the time steps where $\mathcal{G}(t)$ is $2 \mathrm{R}+1$-robust. Therefore, in the $\mathrm{R}$-locally bounded model having faulty nodes, multiscale consensus is reached if $\left|\left\{t_{k}\right\}\right|=\infty$ and there is a constant $c$ satisfying $\left|t_{k+1}-t_{k}\right| \leq c$ for every $k$.

In parallel with Corollary 2, we have the following result for resilient multiscale formation generation under the locally bounded model.

Corollary 4 (formation generation in locally bounded model with faulty nodes). Consider a time-invariant network characterized by a digraph $\mathcal{G}=(\mathcal{V}, \mathcal{E})$, where every cooperative node updates its value following the multiscale filtering-formation strategy with parameter $\mathrm{R}$. Therefore, in the R-locally bounded model containing faulty nodes, multiscale formation $g$ is achieved if $\mathcal{G}$ is $2 \mathrm{R}+1$-robust. Moreover, $\mathcal{G}$ is $\mathrm{R}+1$-robust if multiscale formation $\mathrm{g}$ in the $\mathrm{R}$-locally bounded model with faulty nodes is achieved.

For time-dependent network $\mathcal{G}(t)=(\mathcal{V}, \mathcal{E}(t))$, let $\left\{t_{k}\right\}$ be the time steps where $\mathcal{G}(t)$ is $(\mathrm{R}+1, \mathrm{R}+1)$-robust. Therefore, in the R-locally bounded model having faulty nodes, multiscale formation $g$ is reached if $\left|\left\{t_{k}\right\}\right|=\infty$ and there is a constant $c$ satisfying $\left|t_{k+1}-t_{k}\right| \leq c$ for any $k$.

\subsection{Multiscale Coordination in the Presence of Byzantine Nodes}

In this subsection, we establish necessary and sufficient conditions for multiscale coordination in the globally and locally bounded model in the presence of Byzantine nodes. Recall that Byzantine nodes are able to forward different information to different neighbors at any time step, and thus they are harder to deal with. Define $\mathcal{G}_{\mathcal{C}}(t)=\left(\mathcal{C}, \mathcal{E}_{\mathcal{C}}(t)\right)$ to be the subnetwork of $\mathcal{G}(t)=(\mathcal{V}, \mathcal{E}(t))$ induced by $\mathcal{C}$, where $\mathcal{E}_{\mathcal{C}}(t)$ is made up of all directed edges among the cooperative nodes at time step $t$. Time-invariant network structure will be investigated first. 
Theorem 3 (consensus in globally bounded model with Byzantine nodes). Consider a time-invariant network characterized by a digraph $\mathcal{G}=(\mathcal{V}, \mathcal{E})$, in which every cooperative node updates its value following the multiscale filtering strategy with parameter R. Therefore, in the R-globally bounded model having Byzantine nodes, multiscale consensus is reached if and only if $\mathcal{G}_{\mathcal{C}}$ is $\mathrm{R}+1$-robust.

Proof. (Necessity) Suppose that $\mathcal{G}_{\mathcal{C}}$ is not $\mathrm{R}+1$-robust. Therefore, there exist two nonempty and disjoint sets $S_{1}, S_{2} \subseteq \mathcal{C}$ which are not $\mathrm{R}+1$ reachable. Thereby, every node in these two sets has no more than $\mathrm{R}$ cooperative neighbors outside the set. Fix $a<b$. Let $x_{i}(0)=\frac{a}{\alpha_{i}(0)}$ for any node $v_{i} \in S_{1}$, and $x_{i}(0)=\frac{b}{\alpha_{i}(0)}$ for any node $v_{i} \in S_{2}$. For $v_{i} \in \mathcal{V} \backslash\left\{S_{1} \cup S_{2}\right\}$, set $x_{i}(0)=\frac{c}{\alpha_{i}(0)}$ for some fixed $c \in(a, b)$. Suppose that all Byzantine nodes always send the value $\frac{a}{\alpha_{i}(t)}$ to every node $v_{i}$ in $S_{1}$, and the value $\frac{b}{\alpha_{i}(t)}$ to every node $v_{i}$ in $S_{2}$ at every time step $t$. Therefore, utilizing the multiscale filtering strategy having parameter $\mathrm{R}$, nodes in $S_{1}$ and $S_{2}$ will not adopt values from outside their own sets. Thus, multiscale consensus can not be reached.

(Sufficiency) Similarly, we suppose that $\rho_{M}:=\lim _{t \rightarrow \infty} M(t)$ and $\rho_{m}:=\lim _{t \rightarrow \infty} m(t)$. Assume that $\rho_{M}>\rho_{m}$. Choose $\varepsilon_{0}>0$ satisfying $\rho_{M}-\varepsilon_{0}>\rho_{m}+\varepsilon_{0}$. For $t \in \mathbb{N}$ and $\varepsilon_{k}>0$, we define two sets $B_{M}\left(t, \varepsilon_{k}\right):=\left\{v_{i} \in \mathcal{C}: \alpha_{i}(t) x_{i}(t)>\rho_{M}-\varepsilon_{k}\right\}$ and $B_{m}\left(t, \varepsilon_{k}\right):=\left\{v_{i} \in \mathcal{C}: \alpha_{i}(t) x_{i}(t)<\rho_{m}+\varepsilon_{k}\right\}$. As per the definition of $\varepsilon_{0}, B_{M}\left(t, \varepsilon_{0}\right)$ and $B_{m}\left(t, \varepsilon_{0}\right)$ are disjoint. Fix $\varepsilon<\frac{\alpha^{|\mathcal{C}|} \varepsilon_{0}}{1-\alpha^{|\mathcal{C}|}}$ which satisfies $\varepsilon_{0}>\varepsilon>0$. Define $t_{\varepsilon}$ as the time step satisfying $M(t)<\rho_{M}+\varepsilon$ and $m(t)>\rho_{m}-\varepsilon$ for every time step $t \geq t_{\varepsilon}$.

Recall that $B_{M}\left(t_{\varepsilon}, \varepsilon_{0}\right)$ and $B_{m}\left(t_{\varepsilon}, \varepsilon_{0}\right)$ are nonempty and disjoint. As $\mathcal{G}_{\mathcal{C}}$ is $\mathrm{R}+1$-robust with at most R Byzantine nodes, there exists a node in $B_{M}\left(t_{\varepsilon}, \varepsilon_{0}\right)$ or $B_{m}\left(t_{\varepsilon}, \varepsilon_{0}\right)$ that has more than or equal to $R+1$ cooperative neighboring nodes outside of its set. Without loss of generality, we assume that $v_{i} \in B_{M}\left(t_{\varepsilon}, \varepsilon_{0}\right)$ has more than or equal to $\mathrm{R}+1$ cooperative neighboring nodes outside of $B_{M}\left(t_{\varepsilon}, \varepsilon_{0}\right)$. With the same argument as in Theorem 1 , we derive the inequality $\alpha_{i}\left(t_{\varepsilon}+1\right) x_{i}\left(t_{\varepsilon}+1\right) \leq$ $\rho_{M}-\alpha \varepsilon_{0}+(1-\alpha) \varepsilon$. This expression also holds true for the renewed value of every cooperative node outside $B_{M}\left(t_{\varepsilon}, \varepsilon_{0}\right)$. Likewise, if $v_{i} \in B_{m}\left(t_{\varepsilon}, \varepsilon_{0}\right)$ which has more than or equal to $\mathrm{R}+1$ cooperative neighbors outside of $B_{m}\left(t_{\varepsilon}, \varepsilon_{0}\right)$, we have similarly $\alpha_{i}\left(t_{\varepsilon}+1\right) x_{i}\left(t_{\varepsilon}+1\right) \geq \rho_{m}+\alpha \varepsilon_{0}-(1-\alpha) \varepsilon$, which also applies to the cooperative nodes outside $B_{m}\left(t_{\varepsilon}, \varepsilon_{0}\right)$.

Define $\varepsilon_{1}=\alpha \varepsilon_{0}-(1-\alpha) \varepsilon$, satisfying $0<\varepsilon<\varepsilon_{1}<\varepsilon_{0}$. Notice that the sets $B_{M}\left(t_{\varepsilon}+1, \varepsilon_{1}\right)$ and $B_{m}\left(t_{\varepsilon}+1, \varepsilon_{1}\right)$ are disjoint. The discussion in the above paragraph implies that $\left|B_{M}\left(t_{\varepsilon}+1, \varepsilon_{1}\right)\right|<$ $\left|B_{M}\left(t_{\varepsilon}, \varepsilon_{0}\right)\right|$ or $\left|B_{m}\left(t_{\varepsilon}+1, \varepsilon_{1}\right)\right|<\left|B_{m}\left(t_{\varepsilon}, \varepsilon_{0}\right)\right|$ holds. We can recursively define $\varepsilon_{k}=\alpha \varepsilon_{k-1}-(1-\alpha) \varepsilon$ for every $k \geq 1$ and note that $\varepsilon_{k}<\varepsilon_{k-1}$. The aforementioned discussion is applicable to every time step $t_{\varepsilon}+k$ provided $B_{M}\left(t_{\varepsilon}+k, \varepsilon_{k}\right)$ and $B_{m}\left(t_{\varepsilon}+k, \varepsilon_{k}\right)$ are non-empty. Since $\mathcal{G}_{\mathcal{C}}$ contains $|\mathcal{C}|$ cooperative nodes, there is some $T \leq|\mathcal{C}|$ satisfying either $B_{M}\left(t_{\varepsilon}+T, \varepsilon_{T}\right)$ or $B_{m}\left(t_{\varepsilon}+T, \varepsilon_{T}\right)$ is in fact empty. On the other hand, $\varepsilon_{T}=\alpha \varepsilon_{T-1}-(1-\alpha) \varepsilon=\alpha^{T} \varepsilon_{0}-\left(1-\alpha^{T}\right) \varepsilon \geq \alpha^{|\mathcal{C}|} \varepsilon_{0}-\left(1-\alpha^{|\mathcal{C}|}\right) \varepsilon>0$ according to the choice of $\varepsilon$. This implies that every cooperative node at time $t_{\varepsilon}+T$ has a value of no more than $\rho_{M}-\varepsilon_{T}<\rho_{M}$ or have values no less than $\rho_{m}+\varepsilon_{T}>\rho_{m}$. This contradicts the definition of $\rho_{M}$ or $\rho_{m}$. We proved the sufficiency part.

In the case of time-dependent networks, the following result can be established.

Corollary 5. Consider a time-dependent network characterized by a digraph $\mathcal{G}(t)=(\mathcal{V}, \mathcal{E}(t))$, where every cooperative node updates its value following the multiscale filtering strategy with parameter $R$. Let $\left\{t_{k}\right\}$ be the time steps where $\mathcal{G}(t)$ is $2 \mathrm{R}+1$-robust. Therefore, in the $\mathrm{R}$-globally bounded model having Byzantine nodes, multiscale consensus is reached if $\left|\left\{t_{k}\right\}\right|=\infty$ and there is a constant $c$ satisfying $\left|t_{k+1}-t_{k}\right| \leq c$ for any $k$.

Proof. If $\mathcal{G}(t)$ becomes $2 \mathrm{R}+1$-robust, $\mathcal{G}_{\mathcal{C}}(t)$ must be $\mathrm{R}+1$ robust. It is due to the fact that there exist no more than $R$ Byzantine nodes in the whole network. In view of Theorem 3, we may conclude the proof by resorting to a similar argument as that in Corollary 1 .

The following result is given for resilient multiscale formation generation under the globally bounded model. 
Corollary 6 (formation generation in globally bounded model with Byzantine nodes). Consider a time-invariant network characterized by a digraph $\mathcal{G}=(\mathcal{V}, \mathcal{E})$, in which every cooperative node updates its value following the multiscale filtering-formation strategy with parameter $\mathrm{R}$. Therefore, in the R-globally bounded model containing Byzantine nodes, multiscale formation $g$ is achieved if and only if $\mathcal{G}_{\mathcal{C}}$ is $\mathrm{R}+1$-robust.

For a time-dependent network $\mathcal{G}(t)=(\mathcal{V}, \mathcal{E}(t))$, let $\left\{t_{k}\right\}$ signify the time steps where $\mathcal{G}(t)$ is $2 \mathrm{R}+$ 1-robust. Therefore, in the R-globally bounded model having Byzantine nodes, multiscale formation $g$ is reached if $\left|\left\{t_{k}\right\}\right|=\infty$ and there exists a constant $c$ satisfying $\left|t_{k+1}-t_{k}\right| \leq c$ for all $k$.

Now we move to locally bounded models having Byzantine nodes distributed over a time-invariant graph structure.

Theorem 4 (consensus in locally bounded model with Byzantine nodes). Consider a time-invariant network characterized by a digraph $\mathcal{G}=(\mathcal{V}, \mathcal{E})$, where every cooperative node updates its value following the multiscale filtering strategy with parameter $\mathrm{R}$. Therefore, in the R-locally bounded model containing Byzantine nodes, multiscale consensus is reached if and only if $\mathcal{G}_{\mathcal{C}}$ is $\mathrm{R}+1$-robust.

Proof. (Necessity) The same proof in Theorem 3 gives the necessity.

(Sufficiency) Proceeding similarly as in Theorem 3, we suppose that $\rho_{M}:=\lim _{t \rightarrow \infty} M(t)$ and $\rho_{m}:=\lim _{t \rightarrow \infty} m(t)$. Assume that $\rho_{M}>\rho_{m}$. Choose $\varepsilon_{0}>0$ satisfying $\rho_{M}-\varepsilon_{0}>\rho_{m}+\varepsilon_{0}$. For $t \in \mathbb{N}$ and $\varepsilon_{k}>0$, we signify two sets $B_{M}\left(t, \varepsilon_{k}\right):=\left\{v_{i} \in \mathcal{C}: \alpha_{i}(t) x_{i}(t)>\rho_{M}-\varepsilon_{k}\right\}$ and $B_{m}\left(t, \varepsilon_{k}\right):=\left\{v_{i} \in\right.$ $\left.\mathcal{C}: \alpha_{i}(t) x_{i}(t)<\rho_{m}+\varepsilon_{k}\right\}$. By the definition of $\varepsilon_{0}, B_{M}\left(t, \varepsilon_{0}\right)$ and $B_{m}\left(t, \varepsilon_{0}\right)$ turn out to be disjoint. Fix $\varepsilon<\frac{\alpha|\mathcal{C}|_{\mathcal{E}_{0}}}{1-\alpha|\mathcal{C}|}$ satisfying $\varepsilon_{0}>\varepsilon>0$. Let $t_{\varepsilon}$ be the time step satisfying $M(t)<\rho_{M}+\varepsilon$ and $m(t)>\rho_{m}-\varepsilon$ for any time step $t \geq t_{\varepsilon}$.

Recall that the sets $B_{M}\left(t_{\varepsilon}, \varepsilon_{0}\right)$ and $B_{m}\left(t_{\varepsilon}, \varepsilon_{0}\right)$ are both nonempty and disjoint. As $\mathcal{G}_{\mathcal{C}}$ is $\mathrm{R}+1$-robust, there must be a node in $B_{M}\left(t_{\varepsilon}, \varepsilon_{0}\right)$ or $B_{m}\left(t_{\varepsilon}, \varepsilon_{0}\right)$ that has more than or an equal number of $\mathrm{R}+1$ cooperative neighboring nodes outside of its set. We suppose, without loss of generality, that $v_{i} \in$ $B_{M}\left(t_{\varepsilon}, \varepsilon_{0}\right)$ has more than or equal to $\mathrm{R}+1$ cooperative neighbors outside of $B_{M}\left(t_{\varepsilon}, \varepsilon_{0}\right)$. Since there are no more than $\mathrm{R}$ Byzantine nodes in $\mathcal{N}_{i}, v_{i}$ will adopt at least one of its cooperative neighbors' values outside $B_{M}\left(t_{\varepsilon}, \varepsilon_{0}\right)$ under the multiscale filtering strategy with parameter R. Based upon the same argument as in Theorem 1, we derive the estimation $\alpha_{i}\left(t_{\varepsilon}+1\right) x_{i}\left(t_{\varepsilon}+1\right) \leq \rho_{M}-\alpha \varepsilon_{0}+$ $(1-\alpha) \varepsilon$. This also holds true for the renewed value of every cooperative node outside $B_{M}\left(t_{\varepsilon}, \varepsilon_{0}\right)$. Analogously, if $v_{i} \in B_{m}\left(t_{\varepsilon}, \varepsilon_{0}\right)$ which has more than or an equal number of $\mathrm{R}+1$ cooperative neighbors outside of $B_{m}\left(t_{\varepsilon}, \varepsilon_{0}\right)$, we have $\alpha_{i}\left(t_{\varepsilon}+1\right) x_{i}\left(t_{\varepsilon}+1\right) \geq \rho_{m}+\alpha \varepsilon_{0}-(1-\alpha) \varepsilon$, which also applies to the cooperative nodes outside $B_{m}\left(t_{\varepsilon}, \varepsilon_{0}\right)$.

Define $\varepsilon_{1}=\alpha \varepsilon_{0}-(1-\alpha) \varepsilon$, satisfying $0<\varepsilon<\varepsilon_{1}<\varepsilon_{0}$. The same reasoning in the proof of Theorem 3 (by recursively defining $\varepsilon_{k}=\alpha \varepsilon_{k-1}-(1-\alpha) \varepsilon$ for $k \geq 1$ ) gives rise to the desired contradiction. We then proved the sufficiency part.

For time-dependent networks, the following result can be established. The proof is similar to that of Corollary 5.

Corollary 7. Consider a time-dependent network characterized by a digraph $\mathcal{G}(t)=(\mathcal{V}, \mathcal{E}(t))$, in which every cooperative node updates its value following the multiscale filtering strategy with parameter $\mathrm{R}$. Let $\left\{t_{k}\right\}$ be the time steps where $\mathcal{G}(t)$ is $2 \mathrm{R}+1$-robust. Therefore, in the $\mathrm{R}$-locally bounded model containing Byzantine nodes, multiscale consensus is reached if $\left|\left\{t_{k}\right\}\right|=\infty$ and there is a constant $c$ satisfying $\left|t_{k+1}-t_{k}\right| \leq c$ for any $k$.

As an immediate consequence of Theorem 4 and Corollary 7, resilient multiscale formation generation in the locally bounded model containing Byzantine nodes is characterized in the following.

Corollary 8 (formation generation in locally bounded model with Byzantine nodes). Consider a time-invariant network characterized by a digraph $\mathcal{G}=(\mathcal{V}, \mathcal{E})$, in which every cooperative node updates its 
value following the multiscale filtering-formation strategy with parameter $\mathrm{R}$. Therefore, in the R-locally bounded model with Byzantine nodes, multiscale formation $g$ is reached if and only if $\mathcal{G}_{\mathcal{C}}$ is $\mathrm{R}+1$-robust.

For a time-dependent network $\mathcal{G}(t)=(\mathcal{V}, \mathcal{E}(t))$, let $\left\{t_{k}\right\}$ be the time steps where $\mathcal{G}(t)$ is $2 \mathrm{R}+1$-robust. Therefore, in the R-locally bounded model having Byzantine nodes, multiscale formation $g$ is reached if $\left|\left\{t_{k}\right\}\right|=\infty$ and there exists a constant $c$ satisfying $\left|t_{k+1}-t_{k}\right| \leq c$ for each $k$.

\section{Numerical Simulations}

In this section, we present a range of numerical examples (including two energy systems related ones) to illustrate our results.

Example 1 (Consensus against a single faulty node). We investigate a $(2,2)$-robust network $\mathcal{G}=(\mathcal{V}, \mathcal{E})$ with the node set $\mathcal{V}=\left\{v_{1}, \cdots, v_{6}\right\}$ (see Figure 1 ), in which node $v_{2}$ is compromised and becomes an adversarial node. The initial values of the six agents are taken as $x_{1}(0)=-2, x_{2}(0)=2, x_{3}(0)=3, x_{4}(0)=-1$, $x_{5}(0)=0, x_{6}(0)=1.5$, and the scales are chosen as $\alpha_{1}(t)=\alpha_{2}(t)=\alpha_{3}(t)=2+\sin (t / 5), \alpha_{4}(t)=\alpha_{5}(t)=$ $\alpha_{6}(t)=-1$.

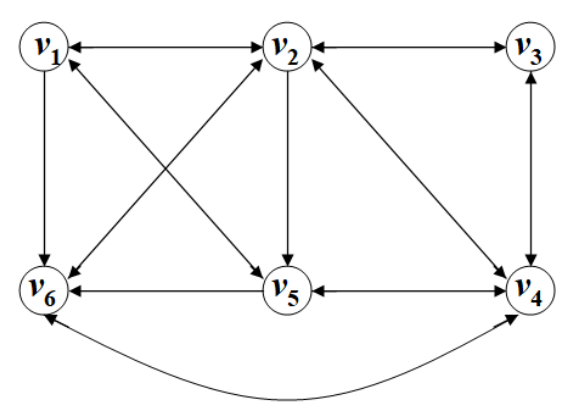

Figure 1. A directed graph $\mathcal{G}$ with $n=6$ nodes, which is $(2,2)$-robust.

Since the network is $(2,2)$-robust, Theorem 1 (or Theorem 2) implies that multiscale consensus can be reached in the 1-globally bounded model with faulty nodes when we implement the multiscale filtering strategy with parameter 1 . We assume that each cooperative node $v_{i} \in \mathcal{C}$ takes the weight $w_{i j}(t)=\left(\left|\alpha_{i}(t)\right|\left(\left|\mathcal{N}_{i}\right|+1-\left|\mathcal{R}_{i}(t)\right|\right)\right)^{-1}$ for $v_{j} \in\left(\mathcal{N}_{i} \cup\left\{v_{i}\right\}\right) \backslash \mathcal{R}_{i}(t)$. In Figure 2a, we show the trajectories of the agents, where the node $v_{2}$ is faulty and keeps its value unchanged. In Figure $2 \mathrm{~b}$, we consider a different situation, where $v_{2}$ is more malicious (aiming to drive the states of the system to perhaps unsafe regions) and updates its value following $x_{2}(t+1)=\left(x_{1}(t)+x_{3}(t)+x_{4}(t)+\right.$ $\left.x_{6}(t)\right) / 4+\mathrm{t} / 10$. We see that, in both cases, the cooperative nodes are able to reach multiscale consensus with respect to $\left(\alpha_{1}(t), \cdots, \alpha_{6}(t)\right)$ as supported by Theorem 1 .

Note that the induced network $\mathcal{G}_{\mathcal{C}}$ is not 2-robust (by considering, e.g., the disjoint atom sets $\left\{v_{1}\right\}$ and $\left.\left\{v_{3}\right\}\right)$. Recall that a faulty node is also Byzantine. We clarify that the multiscale consensus behaviors observed here do not contradict Theorem 3 because Theorem 3 indicates that there exists one specific node that can be compromised to prevent multiscale consensus if $\mathcal{G}_{\mathcal{C}}$ is not 2-robust. Furthermore, it is also worthwhile to note that, although multiscale consensus is achieved in both Figure 2a,b, the adversarial node $v_{2}$ indeed affects the final trajectories of the cooperative nodes. This is allowed in our multiscale coordination framework because the value $x_{i}(t)$ for each cooperative node $v_{i}$ still lies in the interval $\left[m(0) / \alpha_{i}(t), M(0) / \alpha_{i}(t)\right]$ that is kept by cooperative nodes at all time step $t$. 


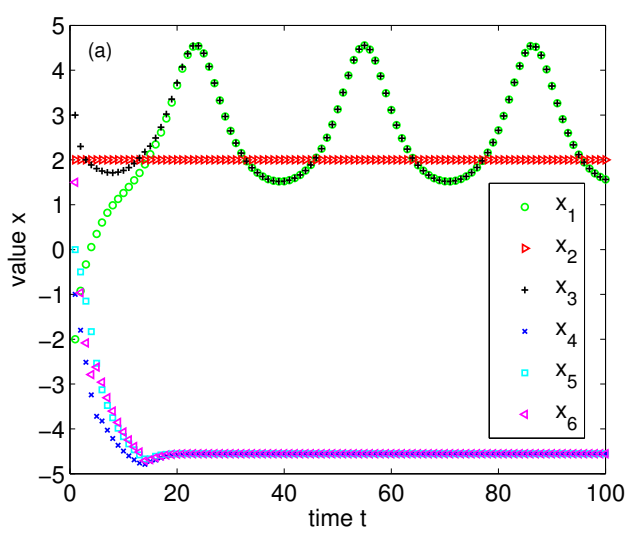

(a)

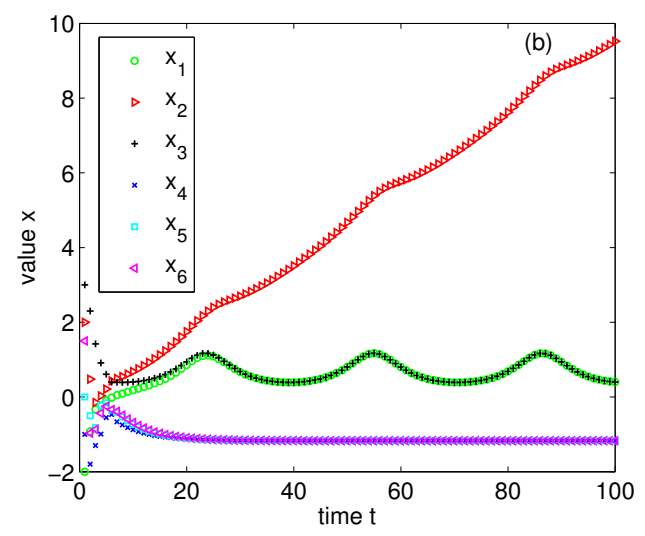

(b)

Figure 2. Time evolution of the agents' values: (a) $v_{2}$ maintains its value and (b) $v_{2}$ updates its value according to $x_{2}(t+1)=\left(x_{1}(t)+x_{3}(t)+x_{4}(t)+x_{6}(t)\right) / 4+t / 10$.

Example 2 (Consensus against a single Byzantine node). In this example, we consider the network topology used in Example 1 except that all communications are assumed to be bidirectional. Moreover, we suppose that $v_{3}$ is compromised (Byzantine) instead. Therefore, the cooperative node set is $\mathcal{C}=\left\{v_{1}, v_{2}, v_{4}, v_{5}, v_{6}\right\}$ and the induced network $\mathcal{G}_{\mathcal{C}}$ becomes 2-robust. The same scales, initial values, and weights for the system are taken as in Example 1. To specify the Byzantine behavior of $v_{3}$, we set $x_{3}(t) \equiv 3, x_{3}^{2}(t) \equiv 2$, and $x_{3}^{4}(t)=2 x_{4}(t)$.

Figure 3 shows the trajectories of the agents reaching multiscale consensus in good agreement with the theoretical results in Theorems 3 and 4 using the multiscale filtering strategy with parameter $\mathrm{R}=1$. Comparing Figure 3 with Figure 2, it is interesting to see that the states of the cooperative agents converge faster in Example 2: multiscale consensus is achieved well before $t=10$ in Figure 3 but achieved only around $t=20$ in Figure $2 \mathrm{a}, \mathrm{b}$. The more rapid consensus presumably results from (i) the higher density in the communication network; and (ii) the less connected compromised node $v_{3}$ in Example 2 as compared to $v_{2}$ in Example 1. In fact, the size of the neighborhood $\mathcal{N}_{2}=\left\{v_{1}, v_{3}, v_{4}, v_{5}, v_{6}\right\}$ nearly triples that of $\mathcal{N}_{3}=\left\{v_{2}, v_{4}\right\}$, which appears to be positively correlated to the convergence time regardless of the particular behavior (viz. faulty or Byzantine) of the respective adversarial node. This unravels that $v_{2}$ should be better protected, for example, in an infrastructure network, due to its central location in the network structure. A detailed investigation of the influence of node location, with certain quantative characterization such as node centralities [29], is likely to reveal more insightful results.

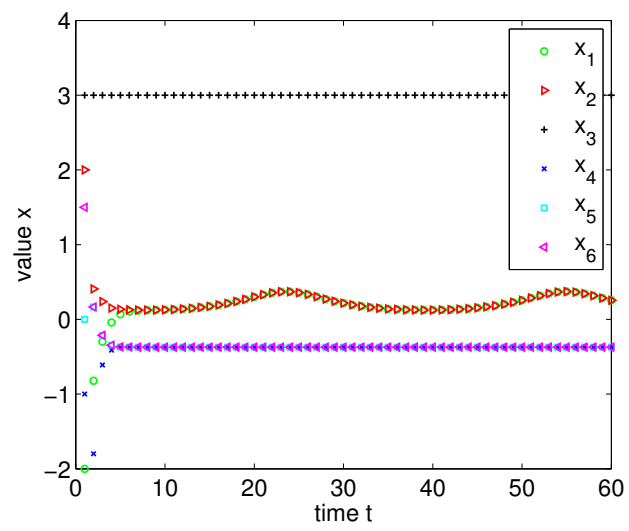

Figure 3. Time evolution of the agents' values, where $v_{3}$ forwards different values to its neighbors, viz. $x_{3}^{2}(t)=2$ and $x_{3}^{4}(t)=2 x_{4}(t)$. 
Example 3 (Consensus against multiple faulty nodes). Here, we reinforce the network in Figure 1 by adding edges $\left\{v_{1}, v_{4}\right\},\left\{v_{1}, v_{3}\right\},\left\{v_{3}, v_{5}\right\}$ and assume that all communications are bidirectional. The resulting network is sketched in Figure 4. It is direct to check that this network is $(3,3)$-robust.

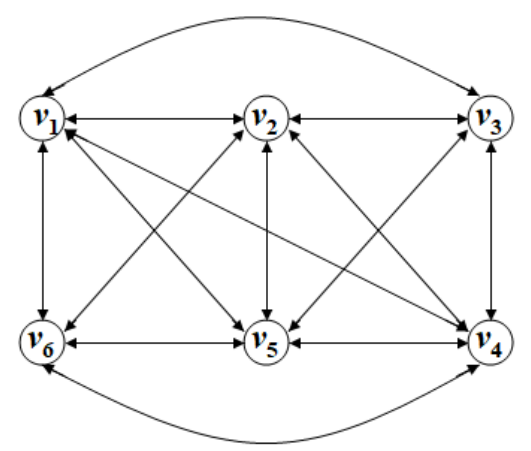

Figure 4. A graph $\mathcal{G}$ with $n=6$ nodes, which is $(3,3)$-robust.

We assume that two highly connected nodes $v_{2}$ and $v_{4}$ are compromised, posing a severe threat to the system performance. Namely, $\mathcal{A}=\left\{v_{2}, v_{4}\right\}$ and $\mathcal{C}=\left\{v_{1}, v_{3}, v_{5}, v_{6}\right\}$. The same scales, initial values, and weights for the system are taken as in Example 1. The state update rules for $v_{2}$ and $v_{4}$ are described by $x_{2}(t+1)=2+t / 20$ and $x_{4}(t+1)=-1-t / 20$, respectively. We perform the multiscale filtering strategy with parameter $R=2$ as per Theorem 1 . As expected, we observe from Figure 5 that the desired multiscale consensus is finally reached in spite of the interference of these two well-connected faulty agents.

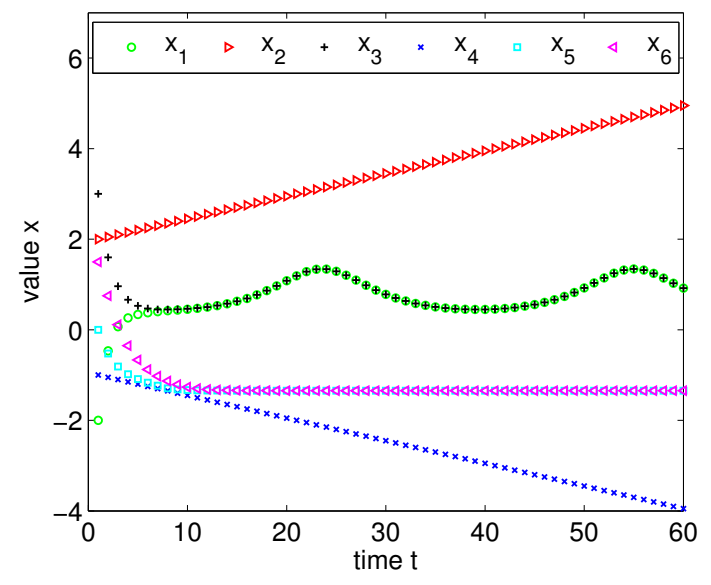

Figure 5. Time evolution of the agents' values, where $v_{2}$ and $v_{4}$ are faulty nodes following $x_{2}(t+1)=$ $2+t / 20$ and $x_{4}(t+1)=-1-t / 20$.

Example 4 (Distributed battery power consensus on a modular microgrid). In this example, we adopt our multiscale consensus theory to realize the power and capacity consensus of battery storage based upon a project located on DongAo Island in China [26]. The traditional power system on islands takes the form of diesel generators which often cause some air pollution and noises. To overcome these drawbacks and improve networking, some stand-alone modular microgrids based on decentralized batteries have been proposed, which essentially entail distributed power and capacity consensus control protocols. The structure of the battery storage system on DongAo Island can be abstracted as an undirected network on four modules, with each agent representing a module; see Figure 6. This network has a (2,2)-robust architecture. 

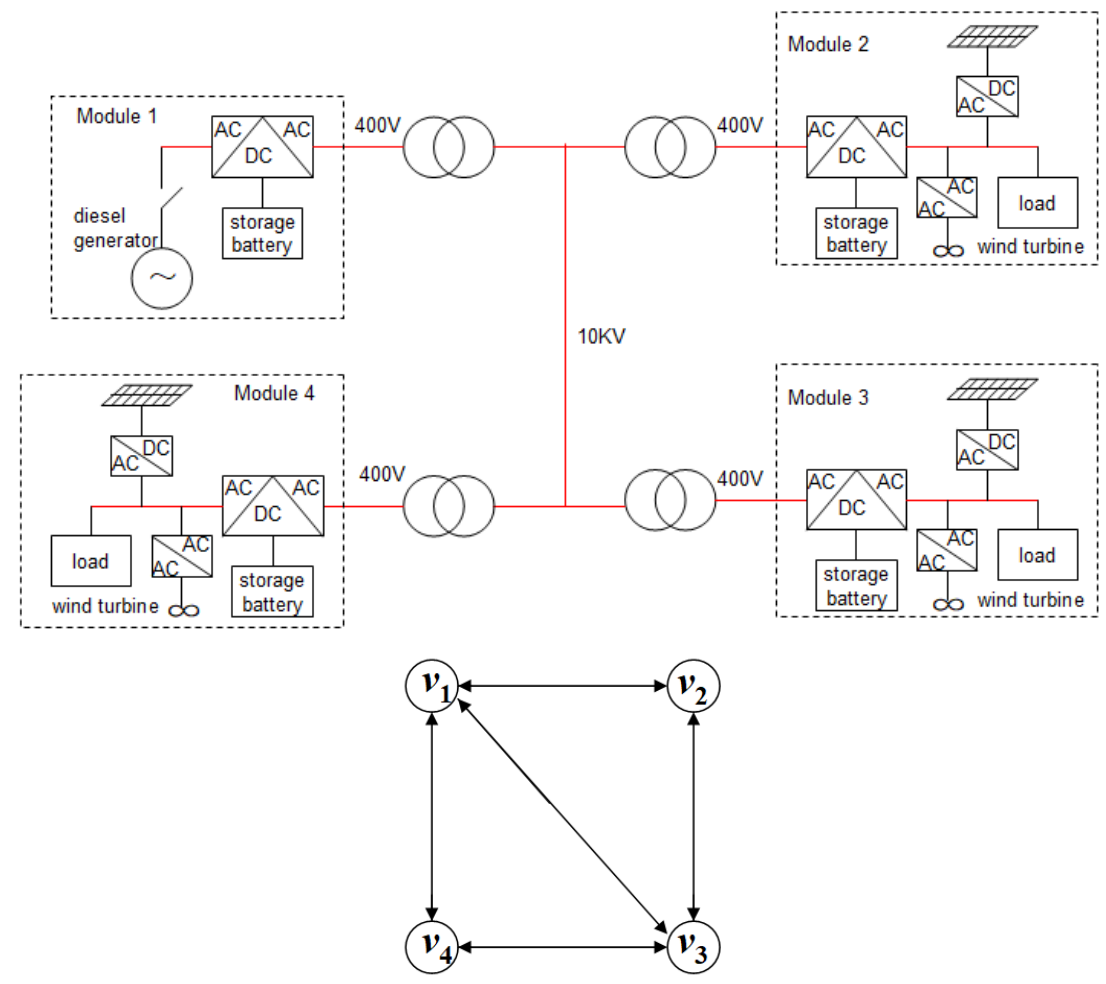

Figure 6. Above: structure of modular microgrid on DongAo Island; Below: communication network $\mathcal{G}$, which is $(2,2)$-robust.

The initial values (battery power) of the four agents are taken as $x_{1}(0)=1, x_{2}(0)=2, x_{3}(0)=3$, $x_{4}(0)=4$, and the scales are chosen to be $\alpha_{1}(t)=\alpha_{2}(t)=1, \alpha_{3}(t)=\alpha_{4}(t)=2$. Let $g=(1,1,1,1)$. We assume that module 2 failed due to some technical error at time $t=20$, namely, $x_{2}(t)=0$ for $t \geq 20$. We plot the power versus time in Figure 7 by using the multiscale formation generation scheme (6) and the multiscale filtering-formation strategy with parameter 1. The results in Figure 7 agree with Corollary 2 . The remaining three functional modules $\left(v_{1}, v_{3}\right.$, and $\left.v_{4}\right)$ managed to achieve the desired multiscale formation and hence the consensus reaching process is robust against adversarial events.

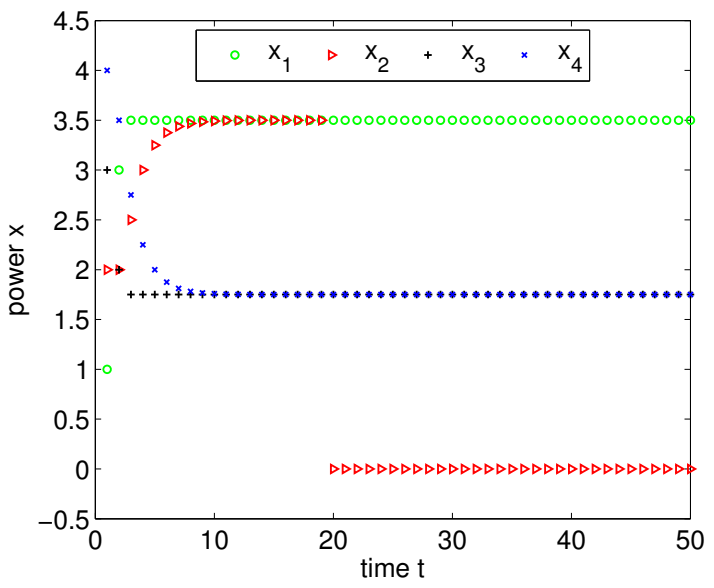

Figure 7. Battery power in modules versus time, where $v_{2}$ breaks down at time $t=20$.

Example 5 (Distributed frequency regulation in an electrical power network). In a smart power grid, frequency regulation service provided by appropriate control of generation is essential for enhancing reliability 
and efficiency of its performance. Each component in the network is coordinated in a distributed manner to provide active power for the provision of ancillary frequency regulations [30]. Here, we consider the decentralized frequency control problem in the presence of a faulty component based on our resilient multiscale consensus theory. The power system consisting of four generators and the corresponding communication network describing the exchange of information between generator agents are shown in Figure 8.
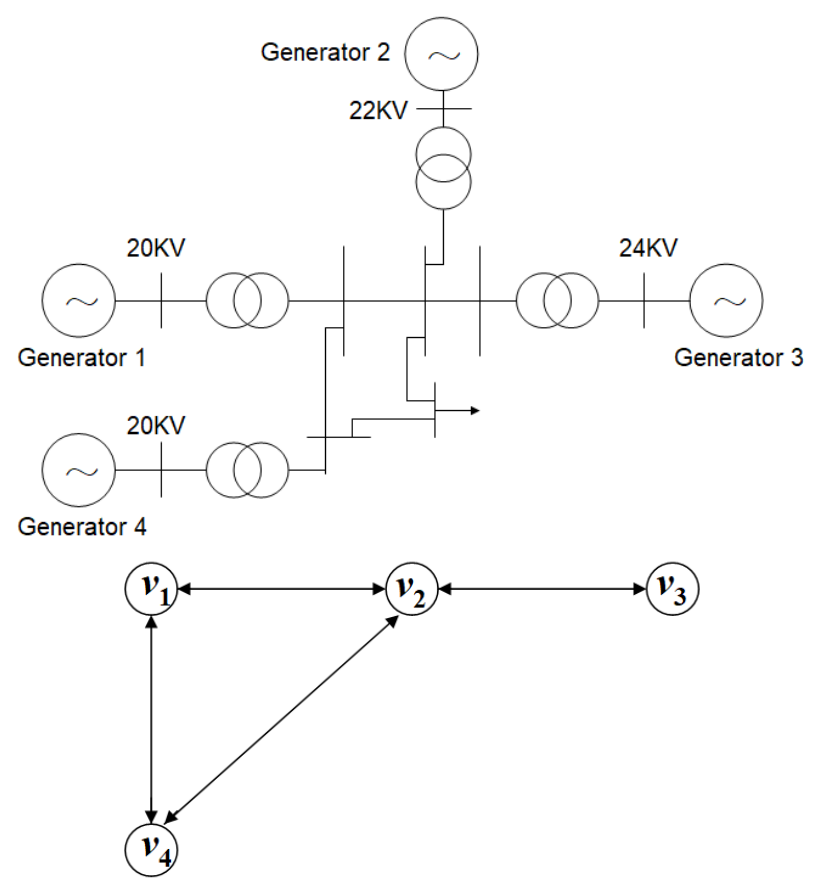

Figure 8. Above: structure of a power system; Below: the topology of a communication network.

We study frequency stability in the presence of a faulty agent represented by $v_{3}$; see Figure 8 . Since $\mathcal{C}=\left\{v_{1}, v_{2}, v_{4}\right\}$, the subnetwork $\mathcal{G}_{\mathcal{C}}$ is 2-robust. The initial values (frequency) of the four agents are taken as $x_{1}(0)=x_{2}(0)=x_{3}(0)=x_{4}(0)=1$, and the scales are chosen to be $\alpha_{i}(t)=i$ for $i=1, \cdots, 4$. Let $g=(1,1,1,1)$. We assume that $x_{3}(t)=1+\sin (t / 5)$. Figure 9 illustrates the evolution of frequency state with respect to time by using the multiscale formation generation scheme (6) and the multiscale filtering-formation strategy with parameter 1 . As one would expect from Corollary 6 , the states of these cooperative agents $\left\{v_{1}, v_{2}, v_{4}\right\}$ reach multiscale consensus withstanding the interference of faulty $v_{3}$.

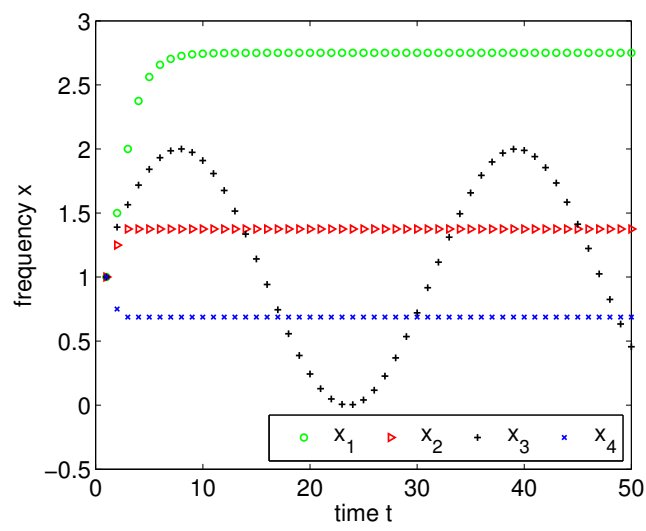

Figure 9. Frequency state versus time, where $v_{3}$ is faulty following $x_{3}(t)=1+\sin (t / 5)$. 


\section{Conclusions}

In this paper, we have investigated resilient multiscale coordination algorithms that are able to withstand the compromise of a subset of nodes in directed networks. We established necessary and sufficient conditions guaranteeing multiscale consensus with general time varying scales in the presence of faulty and Byzantine nodes. Both locally bounded and globally bounded adversaries are dealt with. It is shown that, for a fixed network with at most $R$ faulty nodes, multiscale consensus is achieved if and only if the network is $(R+1, R+1)$-robust. On the other hand, for a network with at most $R$ Byzantine nodes, multiscale consensus is achieved if and only if the induced subnetwork of cooperative nodes is $\mathrm{R}+1$-robust. The theories are then tailored to accommodate time-dependent networks. Multiscale formation generation and formation tracking problems are also solved as the generalizations. We have illustrated the ability to achieve resilient multiscale consensus via extensive numerical examples including the battery storage system on DongAo Island and frequency regulation on power grid. In our future work, we will address distributed update rules that can further accommodate communication delays and external (possibly random) perturbations. Furthermore, extensions of the methods to deal with continuous-time multi-agent systems also seem appealing.

Funding: This work is supported by the National Natural Science Foundation of China under Grant No. 11505127.

Acknowledgments: The author is grateful to the anonymous reviewers for their constructive suggestions that have improved the presentation of the paper.

Conflicts of Interest: The author declares no conflict of interest.

\section{References}

1. Jadbabaie, A.; Lin, J.; Morse, A.S. Coordination of groups of mobile autonomous agents using nearest neighbor rules. IEEE Trans. Autom. Control 2003, 48, 988-1001. [CrossRef]

2. Olfati-Saber, R.; Fax, J.A.; Murray, R.M. Consensus and cooperation in networked multi-agent systems. Proc. IEEE 2007, 95, 215-233. [CrossRef]

3. Cao, Y.; Yu, W.; Ren, W.; Chen, G. An overview of recent progress in the study of distributed multi-agent coordination. IEEE Trans. Ind. Inf. 2013, 9, 427-438. [CrossRef]

4. Ge, X.; Yang, F.; Han, Q.L. Distributed networked control systems: A brief overview. Inf. Sci. 2017, 380, 117-131. [CrossRef]

5. Sun, S.H.; Zhao, L.; Jia, Y.M. Similitude design method for motion reconstruction of space cooperative vehicles. J. Astronaut. 2014, 35, 802-810.

6. Capello, E.; Punta, E.; Dabbene, F.; Guglieri, G.; Tempo, R. Sliding-mode control strategies for rendezvous and docking maneuvers. J. Guid. Control Dyn. 2017, 40, 1481-1487. [CrossRef]

7. Haddad, W.M.; Chellaboina, V.; Hui, Q. Nonnegative and Compartmental Dynamical Systems; Princeton University Press: Princeton, NJ, USA, 2010.

8. Roy, S. Scaled consensus. Automatica 2015, 51, 259-262. [CrossRef]

9. Shang, Y. A combinatorial necessary and sufficient condition for cluster consensus. Neurocomputing 2016, 216, 611-616. [CrossRef]

10. Qin, J.; Fu, W.; Zheng, W.X.; Gao, H. On the bipartite consensus for generic linear multiagent systems with input saturation. IEEE Trans. Cybern. 2017, 47, 948-1958. [CrossRef] [PubMed]

11. Jiang, Y.; Zhang, H.; Chen, J. Sign-consensus of linear multi-agent systems over signed directed graphs. IEEE Trans. Ind. Electron. 2017, 64, 5075-5083. [CrossRef]

12. Meng, D.; Jia, Y. Scaled consensus problems on switching networks. IEEE Trans. Autom. Control 2016, 61, 1664-1669. [CrossRef]

13. Meng, D.; Jia, Y. Robust consensus algorithms for multiscale coordination control of multivehicle systems with disturbances. IEEE Trans. Ind. Electron. 2016, 63, 1107-1119. [CrossRef]

14. Shang, Y. Finite-time scaled consensus through parametric linear iterations. Int. J. Syst. Sci. 2017, 48, 2033-2040. [CrossRef]

15. Shang, Y. On the delayed scaled consensus problems. Appl. Sci. 2017, 7, 713, doi:10.3390/app7070713. [CrossRef] 
16. Lynch, N.A. Distributed Algorithms; Morgan Kaufmann: San Mateo, CA, USA, 1996.

17. Cárdenas, A.A.; Amin, S.; Sastry, S.S. Research challenges for the security of control systems. In Proceedings of the 3rd Conference on Hot Topics in Security, San Jose, CA, USA, 14 April 2008.

18. Zhang, H.; Sundaram, S. Robustness of information diffusion algorithms to locally bounded adversaries. In Proceedings of the 2012 American Control Conference, Montreal, QC, Canada, 27-29 June 2012; pp. 5855-5861.

19. LeBlanc, H.J.; Zhang, H.; Koutsoukos, X.; Sundaram, S. Resilient asymptotic consensus in robust networks. IEEE J. Sel. Areas Commun. 2013, 31, 766-781. [CrossRef]

20. Dibaji, S.M.; Ishii, H. Consensus of second-order multi-agent systems in the presence of locally bounded faults. Syst. Control Lett. 2015, 79, 23-29. [CrossRef]

21. Wu, Y.; He, X. Secure consensus control for multi-agent systems with attacks and communication delays. IEEE/CAA J. Autom. Sin. 2017, 4, 136-142. [CrossRef]

22. Saulnier, K.; Saldaña, D.; Prorok, A.; Pappas, G.J.; Kumar, V. Resilient flocking for mobile robot teams. IEEE Robot. Autom. Lett. 2017, 2, 1039-1046. [CrossRef]

23. Shang, Y. Hybrid consensus for averager-copier-voter networks with non-rational agents. Chaos Solitons Fractals 2018, 110, 244-251. [CrossRef]

24. Trong, T.N.; Duc, M.N. Sliding surface in consensus problem of multi-agent rigid manipulators with neural network controller. Energies 2017, 10, 2127. [CrossRef]

25. Zhang, F.; Leonard, N.E. Cooperative filters and control for cooperative exploration. IEEE Trans. Autom. Control 2010, 55, 650-663. [CrossRef]

26. Zhang, X.; Huang, Y.; Li, L.; Yeh, W.C. Power and capacity consensus tracking of distributed battery storage systems in modular microgrids. Energies 2018, 11, 1439. [CrossRef]

27. Zhang, H.; Fata, E.; Sundaram, S. A notion of robustness in complex networks. IEEE Trans. Control Netw. Syst. 2015, 2, 310-320. [CrossRef]

28. Xiao, F.; Wang, L.; Chen, J.; Gao, Y. Finite-time formation control for multi-agent systems. Automatica 2009, 45, 2605-2611. [CrossRef]

29. Shang, Y. Subgraph robustness of complex networks under attacks. IEEE Trans. Syst. Man Cybern. Syst. 2017. [CrossRef]

30. Kundur, P. Power System Stability and Control, 2nd ed.; McGraw-Hill: New York, NY, USA, 2008.

(C) 2018 by the author. Licensee MDPI, Basel, Switzerland. This article is an open access article distributed under the terms and conditions of the Creative Commons Attribution (CC BY) license (http://creativecommons.org/licenses/by/4.0/). 